Rombouts, K., Bémeur, C., \& Rose, C. F. (2016). Targeting the muscle for the treatment and prevention of hepatic encephalopathy. J ournal of Hepatology, 65(5), 876-878.

The final publication is available at : https://doi.org/10.1016/j.jhep.2016.08.014

Hepatology

Elsevier Editorial System(tm) for Journal of Manuscript Draft

Manuscript Number:

Title: Targeting the muscle for the treatment and prevention of hepatic encephalopathy

Article Type: Invited Editorial

Section/Category: Cirrhosis

Corresponding Author: Dr. Christopher F. Rose, PhD

Corresponding Author's Institution: Université de Montreal, CRCHUM

First Author: Krista Rombouts

Order of Authors: Krista Rombouts; Chantal Bémeur; Christopher F. Rose, $\mathrm{PhD}$ 
1

2

\section{Title: Targeting the muscle for the treatment and prevention of hepatic encephalopathy}

Krista Rombouts ${ }^{1}$, Chantal Bémeur ${ }^{2,3}$, Christopher Rose ${ }^{2}$

${ }^{1}$ University College London UCL, Institute for Liver \& Digestive Health, Royal Free Hospital, London, United Kingdom, ${ }^{2}$ Hepato-Neuro Laboratory, CRCHUM, Université de Montréal, Montreal, Quebec, Canada, ${ }^{3}$ Département de nutrition, Faculté de médecine, Université de Montréal, Montreal, Quebec, Canada.

\section{Authors contributed equally.}

Correspondence:

Christopher Rose, Chantal Bémeur

Hepato-Neuro Laboratory, CRCHUM, 900 Saint-Denis Street, Montreal, Quebec, Canada, H2X 0A9

Phone: 514-890-8000 Ext. 35739

E-mail: christopher.rose@umontreal.ca

E-mail: chantal.bemeur@umontreal.ca

Krista Rombouts PhD. Royal Free Hospital \& University College London, Institute for Liver \& Digestive Health, Division of Medicine, Rowland Hill Street, London, NW3 2PF, United Kingdom Phone: +44 02074332794; Fax: +44 02074332852

E-mail: $\underline{\text { k.rombouts@ucl.ac.uk }}$

Competing interest: none 
Muscle mass loss or sarcopenia is a principle component of malnutrition which prevails in $65-90 \%$ of patients with end-stage liver disease [1]. Intuitively, the roots of malnutrition play a precipitating role in muscle catabolism. Undernutrition frequently occurs in cirrhosis since an inadequate diet is compounded by a hypermetabolic energy demand. However, multiple other factors contribute to the pathogenesis of malnutrition including malabsorption of nutrients, metabolic alterations, increased intestinal protein losses, reduced protein synthesis, increased protein catabolism and disturbance of substrate utilization [2,3]. Sarcopenia adversely affects quality of life, leads to longer hospitalizations, increases susceptibility to infections, negatively impacts clinical outcomes pre- and post-liver transplantation and is an independent prognostic factor for survival in patients with cirrhosis [4]. The pathophysiological pathways triggering a reduction in muscle protein synthesis and/or an increase in proteolysis, resulting in loss of muscle mass, remain elusive. Furthermore, in addition to loss of quantity, quality of muscle is also affected during muscle wasting. Altered muscle metabolism and contractile function, concomitant with a reduction in muscle mass, contributes to the onset of frailty as well as functional decline in physical performance, leading to increased morbidity in patients with cirrhosis [5].

The liver is a powerful metabolic organ which in addition to being the center for the metabolism of nutrients (glucose and lipids), also plays a major role in ammonia disposal. Exclusively expressing all the enzymes of the urea cycle, the liver regulates the circulating blood ammonia levels arising from the gut (primary source of ammonia generation). Thus, severe liver impairment, leads to the occurrence of hyperammonemia. At physiological $\mathrm{pH}$, the majority (98\%) of ammonia is found in ionic form $\left(\mathrm{NH}_{4}{ }^{+}\right)$ with $\sim 2 \%$ arising in gas form $\left(\mathrm{NH}_{3}\right)$. Both forms are capable of crossing cellular membranes. $\mathrm{NH}_{3}$ via diffusion and $\mathrm{NH}_{4}{ }^{+}$via $\mathrm{K}^{+}$-channels and cotransporters since $\mathrm{NH}_{4}{ }^{+}$has very similar ionic properties (ionic radius and diffusion coefficient) to $\mathrm{K}^{+}$. In addition, specific ammonia transporters have also been identified [6]. Subsequently, following its concentration gradient, ammonia is dispersed throughout the 
body, entering all cells, organs and tissues. Elevated concentrations of ammonia (including increased flux (uptake) of ammonia across cell membranes) lead to changes in $\mathrm{pH}$, adjusted membrane potential and altered cell metabolism, which independently and/or collectively lead to a cascade of pathophysiological events [7]. Since ammonia easily crosses the blood-brain barrier, blood-derived ammonia leads to neurotoxic levels of ammonia in the brain which is a fundamental component implicated in the pathogenesis of hepatic encephalopathy (HE).

Aside from primarily affecting the brain, the toxicity of ammonia has also demonstrated to affect other organs and tissues, including muscle. Muscle plays a significant compensatory role in detoxifying ammonia during liver disease since it houses the enzyme glutamine synthetase (GS), an important ammonia removing pathway during the amidation of glutamate to glutamine. Therefore, in the setting of liver disease, reduced capacity to remove ammonia in the liver, aggregated with muscle mass depletion, further reduces the body's capacity to clear ammonia which in turn leads to a higher risk of developing hyperammonemia and HE [8]. However, paradoxically, within the last 5 years, Dasarathy and colleagues have provided solid evidence that elevated levels of ammonia cause detrimental effects to the muscle. It has been shown that elevated ammonia i) upregulates myostatin (an autocrine growth inhibitor) in myotubes through a NF- $\mathrm{BB}-$ dependent pathway [9], ii) stimulates muscle autophagy [10] and iii) impairs skeletal muscle contractility and strength [11]. In the recent issue (JHEPAT-D-1502306R2), Davuluri and colleagues, using human tissue as well as in vitro and in vivo models of hyperammonemia (including knockout and knockdown of a number of molecular targets), elegantly identified the molecular pathways implicated in the inhibition of muscle protein synthesis in patients with cirrhosis and hyperammonemia [12]. The authors found that ammonia activated the general control nonderepressible 2 (GCN2) kinase (amino acid deficiency sensor) which inactivated the eukaryotic initiation factor 2 (eIF $2 \alpha$ ) (via phosphorylation of the $\alpha$ subunit) and additionally inactivated mTORC1, resulting in global repression of mRNA translation and hence protein synthesis in the 
skeletal muscle. Under physiological conditions, phosphorylation of eIF $2 \alpha$ is followed by an adaptive integrated stress response (ISR) that is mediated via upregulation of activating transcription factor 4 (ATF4) which through downstream signalling pathways leads to the reversible dephosphorylation of phospho-eIF2 $\alpha$. Here, authors demonstrated that loss of feedback negative loop of ISR during hyperammonemia, as evidenced by failure in ATF4 induction, results in chronic and persistent low protein synthesis. Since the effects of increased ammonia ( $\mathrm{pH}$, membrane potential and metabolism) touch all cells within the body and therefore, are not specific to the brain, the importance of hyperammonemia and its impact on clinical outcomes merits to be thoroughly investigated.

Davuluri and colleagues also demonstrated in this issue (JHEPAT-D-15-02306R2) the therapeutic benefit of L-leucine on muscle protein synthesis that was due to the metabolic adaptation of muscle to hyperammonemia. Leucine, a branched-chain amino acid (BCAA), is capable of activating mTORC1 and activating protein synthesis [13]. Furthermore, the authors demonstrated that L-leucine reversed GCN2-eIF2 $\alpha$ phosphorylation, removing protein synthesis inhibition. The authors determined that leucine "starvation", provoked by ammonia, resulted in a compensatory upregulation of the leucine transporter (leucine/glutamine exchanger (SLC7A5)) and therefore leucine supplementation rescued the inhibition of protein synthesis.

Davaluri and colleagues administered $15 \mathrm{~g}$ of leucine-enriched BCAA (7.5g L-leucine, 3.75g Lisoleucine and $3.75 \mathrm{~g}$ L-valine) to 6 hyperammonemic patients with cirrhosis and demonstrated a reversal in muscle protein synthesis inhibition. However, muscle mass (quantity or quality) was not evaluated, most likely due to short treatment time of 7 hours. A supporting study by Les et al., 2011 demonstrated leucine-enriched BCAAs (13.5g L-leucine, 9g L-isoleucine and 7.5g L-valine) given as a nutritional supplementation for 56 weeks lead to an improvement in muscle mass (mid-arm muscle circumference) in 46 patients with cirrhosis [14]. To date, only one study has tested the independent effect of L-leucine and, following 10g/day supplementation for 12 weeks to 9 patients with cirrhosis, 
thigh circumference was not enhanced [15]. This study puts in question the beneficial effect of Lleucine on optimizing muscle mass. However, following the positive results of Davuluri and colleagues, a time-dependent, dose-response study evaluating the effect of L-leucine on muscle mass merits to be thoroughly conducted.

The beneficial effect of L-leucine on muscle protein synthesis and subsequently muscle wasting may also be a result of lowering ammonia. BCAAs are highly metabolized in the muscle and are capable of replenishing $\alpha$-ketoglutarate, believed to be depleted during hyperammonemia as $\alpha$ ketoglutarate is aminated to glutamate which subsequently is amidated to glutamine. These ammoniaremoving pathways are stimulated during hyperammonemia in attempt to reduce ammonia. BCAAs have been reported to lower blood ammonia following 3 months of daily supplementation $(0.24 \mathrm{~g} / \mathrm{kg}$; $50 \%$ L-leucine, $25 \%$ isoleucine and $25 \%$ valine) in patients with cirrhosis which was associated with an improvement in HE [16]. The treatment time and dose appear to be vital as 3-hour administration of BCAAs $(0.45 \mathrm{~g} / \mathrm{kg} ; 45.5 \%$ L-leucine, $30 \%$ isoleucine and $24.5 \%$ valine $)$ did not lower blood ammonia in patients with cirrhosis but did increase BCAA-derived ammonia clearance in muscle [17]. It remains to be defined whether the beneficial effect of L-leucine (BCAA) on muscle protein synthesis is due to a lowering of blood ammonia.

Patients with cirrhosis and sarcopenia have a higher risk of developing HE [8] as loss of muscle mass, in addition to liver impairment, further reduces the capacity of ammonia removal, leading to higher risk of hyperammonemia [18]. There is an invested interest in improving extra-hepatic ammonia clearance (targeting GS in the muscle) for the treatment of HE [19]. Both L-ornithine L-aspartate and L-ornithine phenylacetate have demonstrated to lower blood ammonia by generating glutamate and stimulating GS activity in the muscle [20,21]. However, L-ornithine phenylacetate may be more efficient by chelating glutamine released during muscle catabolism and preventing ammonia generation through glutaminase activity. Overall, increasing the muscle's capacity to clear ammonia by either stimulating GS activity and/or optimizing muscle mass are attractive strategies for the treatment and 
prevention of HE. A recent meta-analysis revealed that BCAAs have a beneficial effect on HE [22]; however the precise mechanisms remain unclear. Interestingly, exercise has been shown to be advantageous in optimizing muscle mass in patients with liver disease [23] and furthermore, in combination with L-leucine supplementation, exercise demonstrated to a have greater impact of muscle mass which was associated with an improvement in minimal HE compared to L-leucine alone [15]. The independent effect of exercise on HE has not been evaluated.

In conclusion, the integrated relationship between muscle (mass and activity of GS), ammonia and cognitive function remains to be comprehensively investigated. Studies evaluating the beneficial effect of improving muscle mass on ammonia clearance and the treatment and prevention of HE are lacking. The data presented by Davuluri and colleagues highlights the important contribution of hyperammonemia on the inhibition of muscle protein synthesis and muscle mass wasting in cirrhosis. This depicts the toxicity of ammonia lies beyond the brain and provides compelling evidence that longterm treatment of hyperammonemia can be beneficial for the muscle and the brain in cirrhosis, improving clinical outcomes pre- and post-liver transplantation.

[1] Merli M, Giusto M, Gentili F, Novelli G, Ferretti G, Riggio O, et al. Nutritional status: its influence on the outcome of patients undergoing liver transplantation. Liver Int 2010;30:208-14. doi:10.1111/j.1478-3231.2009.02135.x.

[2] Bémeur C, Desjardins P, Butterworth RF. Role of nutrition in the management of hepatic encephalopathy in end-stage liver failure. J Nutr Metab 2010;2010:489823. doi:10.1155/2010/489823.

[3] Dasarathy S. Cause and management of muscle wasting in chronic liver disease. Curr Opin Gastroenterol 2016;32:159-65. doi:10.1097/MOG.0000000000000261.

[4] Thandassery RB, Montano-Loza AJ. Role of Nutrition and Muscle in Cirrhosis. Curr Treat Options Gastroenterol 2016;14:257-73. doi:10.1007/s11938-016-0093-z.

[5] Lai JC, Dodge JL, Sen S, Covinsky K, Feng S. Functional decline in patients with cirrhosis awaiting liver transplantation: Results from the functional assessment in liver transplantation (FrAILT) study. Hepatology 2016;63:574-80. doi:10.1002/hep.28316. 
[6] Weiner ID, Verlander JW. Molecular physiology of the Rh ammonia transport proteins. Curr Opin Nephrol Hypertens 2010;19:471-7. doi:10.1097/MNH.0b013e32833bfa4e.

[7] Bosoi CR, Rose CF. Identifying the direct effects of ammonia on the brain. Metab Brain Dis 2009;24:95-102. doi:10.1007/s11011-008-9112-7.

[8] Merli M, Giusto M, Lucidi C, Giannelli V, Pentassuglio I, Di Gregorio V, et al. Muscle depletion increases the risk of overt and minimal hepatic encephalopathy: results of a prospective study. Metabolic Brain Disease 2013;28:281-284. doi:10.1007/s11011-012-9365-z.

[9] Qiu J, Thapaliya S, Runkana A, Yang Y, Tsien C, Mohan ML, et al. Hyperammonemia in cirrhosis induces transcriptional regulation of myostatin by an NF- $\kappa \mathrm{B}-$-mediated mechanism. Proc Natl Acad Sci USA 2013;110:18162-7. doi:10.1073/pnas.1317049110.

[10] Qiu J, Tsien C, Thapalaya S, Narayanan A, Weihl CC, Ching JK, et al. Hyperammonemiamediated autophagy in skeletal muscle contributes to sarcopenia of cirrhosis. Am J Physiol Endocrinol Metab 2012;303:E983-993. doi:10.1152/ajpendo.00183.2012.

[11] McDaniel J, Davuluri G, Hill EA, Moyer M, Runkana A, Prayson R, et al. Hyperammonemia results in reduced muscle function independent of muscle mass. Am J Physiol Gastrointest Liver Physiol 2016;310:G163-170. doi:10.1152/ajpgi.00322.2015.

[12] Davuluri G, Krokowski D, Guan B-J, Kumar A, Thapaliya S, Singh D, et al. Metabolic adaptation of skeletal muscle to hyperammonemia drives the beneficial effects of L-leucine in cirrhosis. J Hepatol 2016. doi:10.1016/j.jhep.2016.06.004.

[13] Wilkinson DJ, Hossain T, Hill DS, Phillips BE, Crossland H, Williams J, et al. Effects of leucine and its metabolite $\beta$-hydroxy- $\beta$-methylbutyrate on human skeletal muscle protein metabolism. $\mathbf{J}$ Physiol (Lond) 2013;591:2911-23. doi:10.1113/jphysiol.2013.253203.

[14] Les I, Doval E, García-Martínez R, Planas M, Cárdenas G, Gómez P, et al. Effects of branchedchain amino acids supplementation in patients with cirrhosis and a previous episode of hepatic encephalopathy: a randomized study. Am J Gastroenterol 2011;106:1081-8. doi:10.1038/ajg.2011.9.

[15] Román E, Torrades MT, Nadal MJ, Cárdenas G, Nieto JC, Vidal S, et al. Randomized pilot study: effects of an exercise programme and leucine supplementation in patients with cirrhosis. Dig Dis Sci 2014;59:1966-75. doi:10.1007/s10620-014-3086-6.

[16] Marchesini G, Dioguardi FS, Bianchi GP, Zoli M, Bellati G, Roffi L, et al. Long-term oral branched-chain amino acid treatment in chronic hepatic encephalopathy. A randomized double-blind casein-controlled trial. The Italian Multicenter Study Group. J Hepatol 1990;11:92-101.

[17] Dam G, Keiding S, Munk OL, Ott P, Buhl M, Vilstrup H, et al. Branched-chain amino acids increase arterial blood ammonia in spite of enhanced intrinsic muscle ammonia metabolism in patients with cirrhosis and healthy subjects. Am J Physiol Gastrointest Liver Physiol 2011;301:G269-277. doi:10.1152/ajpgi.00062.2011. 
[18] Damink SO. Interorgan ammonia and amino acid metabolism in metabolically stable patients with cirrhosis and a TIPSS. Hepatology 2002;36:1163-1171. doi:10.1053/jhep.2002.36497.

[19] Rose CF. Ammonia-Lowering Strategies for the Treatment of Hepatic Encephalopathy. Clin Pharmacol Ther 2012;92:321-31. doi:10.1038/clpt.2012.112.

[20] Rose C, Michalak A, Rao KV, Quack G, Kircheis G, Butterworth RF. L-ornithine-L-aspartate lowers plasma and cerebrospinal fluid ammonia and prevents brain edema in rats with acute liver failure. Hepatology 1999;30:636-640. doi:10.1002/hep.510300311.

[21] Ytrebø LM, Kristiansen RG, Maehre H, Fuskevåg OM, Kalstad T, Revhaug A, et al. Lornithine phenylacetate attenuates increased arterial and extracellular brain ammonia and prevents intracranial hypertension in pigs with acute liver failure. Hepatology 2009;50:165-174. doi:10.1002/hep.22917.

[22] Gluud LL, Dam G, Les I, Córdoba J, Marchesini G, Borre M, et al. Branched-chain amino acids for people with hepatic encephalopathy. Cochrane Database Syst Rev 2015;2:CD001939. doi:10.1002/14651858.CD001939.pub2.

[23] Zenith L, Meena N, Ramadi A, Yavari M, Harvey A, Carbonneau M, et al. Eight Weeks of Exercise Training Increases Aerobic Capacity and Muscle Mass and Reduces Fatigue in Patients With Cirrhosis. Clin Gastroenterol Hepatol 2014;12:1920-1926.e2. doi:10.1016/j.cgh.2014.04.016. 